comprehensive assessment for complications of diabetes that included being directly asked whether they had ED (i.e. inability to attain and/or maintain penile erection sufficient for satisfactory sexual intercourse). Patients were followed up for new CHD events (acute myocardial infarction, death due to coronary cause or other nonfatal CHD event) for a median of 4 years.

A total of 616 (26.7\%) men had ED at baseline, and new CHD events occurred in 123 (5.3\%) patients during follow-up. The annualized incidence of new CHD events was higher in men with ED than in those without ED (19.7 vs 9.5 events per 1,000 person-years). ED was an independent predictor of CHD events following adjustment for confounding factors (hazard ratio 1.58, 95\% Cl 1.08-2.30; $P=0.018$ ).

The authors suggest that men with type 2 diabetes should be monitored for signs of ED in order to identify and treat individuals at risk of future CHD.

Original article Ma RC et al. (2008) Erectile dysfunction predicts coronary heart disease in type 2 diabetes. J Am Coll Cardiol 51: 2045-2050

\section{Men with breast cancer have an increased incidence of prostate cancer}

Previous studies have indicated that men with breast cancer have an increased risk of secondary malignancy. Given that steroid hormones are implicated in both breast and prostate cancer, Lee and Jones investigated the incidence of prostate cancer in men with breast cancer.

The authors retrospectively analyzed medical records of all male patients treated at the Cleveland Clinic between January 1990 and August 2006, and identified 69 men who had received treatment for breast cancer.

In total, $12(17 \%)$ of the 69 men with breast cancer were also diagnosed with prostate cancer. Prostate cancer was detected in these 12 patients by PSA screening, which was only carried out in 38 of the patients studied; therefore, the risk of secondary prostate cancer in the screened population was $31.6 \%$. In six cases the diagnosis of breast cancer preceded that of prostate cancer, whereas the diagnosis of prostate cancer was made first in the other six cases. Of the 12 patients with both breast and prostate cancer, 9 breast cancers were estrogen-receptor positive, 5 were progesterone-receptor positive, and 1 was positive for human epidermal growth factor receptor 2 .

The incidence of prostate cancer in this cohort of men is higher than that expected in the general population, which indicates that men with breast cancer are at increased risk of prostate cancer and should, therefore, undergo PSA screening.

Original article Lee UJ and Jones JS (2008) Incidence of prostate cancer in male breast cancer patients: a risk factor for prostate cancer screening. Prostate Cancer Prostatic Dis [doi:10.1038/pcan.2008.26]

\section{Organochlorine pesticides increase risk of germ cell cancers}

Exposure to persistent organochlorine pesticides (POPs) has been associated with an increased risk of testicular germ cell tumors (TGCTs). Now, the US Servicemen's Testicular Tumor Environmental and Endocrine Determinants study has produced evidence that supports the link between POPs and TGCTs.

McGlynn et al. conducted a pair-matched, case-control study that included 739 patients with TGCT and 915 control participants; 685 pairs were matched for age and ethnicity. Of the 685 patients, 300 had seminoma and 385 had nonseminoma TGCTs. Patients were, on average, taller than controls and were more likely to have a history of cryptorchism or family history of testicular cancer.

After adjustment for confounding variables, high serum levels of POPs were associated with an increased risk of TGCT. For serum levels of cis-nonachlor, trans-nonachlor, and total chlordanes, the risk of TGCT was $~ 50 \%$ higher for patients in the highest quartile than for those in the lowest quartile. For $p, p^{\prime}$-dichlorodiphenyldichloroethylene (DDE, a metabolite of dichlorodiphenyltrichloroethane [DDT]), patients in the highest quartile had a $71 \%$ higher risk of TGCT than those in the lowest quartile. Both DDE and chlordanes were associated with an almost $100 \%$ increased risk of seminoma; however, only DDE was associated with increased risk of nonseminoma TGCTs. 